\title{
RF Synthesizer Loop Filter Design for Minimal OFDM Inter-carrier Interference
}

\author{
Vitor Fialho ${ }^{1,2}$, Fernando Azevedo ${ }^{2,3}$, Fernando Fortes ${ }^{2,3}$, and Manuela Vieira ${ }^{1,2}$ \\ ${ }^{1}$ Universidade Nova de Lisboa - Faculdade de Ciências e Tecnologia- DEE. \\ Monte da Caparica 2829-516, Lisbon, Portugal \\ ${ }^{2}$ Instituto Superior de Engenharia de Lisboa - ISEL. \\ Rua Conselheiro Emídio Navarro,1 1959-007-Lisbon, Portugal \\ ${ }^{3}$ Instituto das Telecomunicações \\ Av. Rovisco Pais, 11049 - 001 Lisbon - Portugal \\ \{vfialho, fazevedo, ffortes\}@deetc.isel.ipl.pt, mv@isel.pt
}

\begin{abstract}
This paper describes the influence of Radio-Frequency synthesizer loop filter design on OFDM inter-carrier interference. OFDM modulation scheme is very sensitive on carrier phase and frequency variations, resulting on inter-carrier interference leading to incorrect message decoding and the increase of system bit error rate. Radio-Frequency synthesizer has the main role on modulation and demodulation processes of base band IQ signals. The loop filter is one of its building blocks designed for loop stability. It also shapes the phase noise frequency response at the output of the frequency synthesizer. The achieved results are supported by simulation scenarios based on OFDM architecture, including radio-frequency synthesizer topology. The loop filter frequency response, local oscillator phase noise power and bandwidth are configurable. These simulations features allow the design trade-off between synthesizer stability and output phase noise power.
\end{abstract}

Keywords: Synthesizer, loop filter, phase noise, inter-carrier interference.

\section{Introduction}

Orthogonal frequency division multiplexing (OFDM) is a widely known modulation technique used in several wireless communication standards [1], [2]. OFDM specificity, based on slow sub-channel rate, minimizes the inter-symbolic interference (ISI) and channel multi-path interference [3], [4]. The sub-channel orthogonality is ensured by the inverse fast-Fourier transform (IFFT), where each input element represents the data to be carried on the correspondent sub-channel. However, OFDM is highly sensitive to phase and frequency variations which may cause the loss of orthogonality, leading to incorrect message decoding and, consequently, the increase of system bit error rate (BER) [4]. These effects are mainly caused by the radiofrequency (RF) front-end impairments during frequency conversion.

$\mathrm{RF}$ front-end is the main block between the base-band (BB) processor and the antenna, and its building blocks are responsible for the up and down conversion of 
in-phase (I) and quadrature (Q) BB signals. These operations are performed by the mixer and oscillator. The oscillator used in RF transceivers is embedded in a synthesizer topology to achieve an accurate output frequency, with small and precise variations steps [5]. The need for an output signal with high accuracy implies the use of phase lock loop (PLL)/ synthesizer architecture to obtain a stable local oscillator (LO) signal for the frequency conversion. However, the oscillator in the synthesizer is responsible for the injection of phase noise into it and consequently, through the entire frequency conversion process. Its main effect on OFDM is the loss of channel orthogonality, leading to the interference between the adjacent sub-channels [4]. In OFDM transmission, besides the transmitted data, it is also included carrier pilots which provide the estimation of channel propagation conditions. This is done before the RF frequency conversion, therefore, these pilots will be also affected with phase noise.

Phase noise is a widely studied topic in free run oscillators [6], [7]. When the oscillator is inserted in a synthesizer to obtain the LO, the phase noise spectral behavior is modified [5], [8].

There is some literature addressing OFDM under phase noise influence [8], [9], [10], however these works are based on a classical synthesizer topology, where the presented results are based on synthesizer noise characterization, not addressing the effects of phase noise on the up-converted channel and how to minimize it.

Due to the constant demand for transceiver miniaturization and low power consumptions, the actual RF front-end, namely RF synthesizer, is totally based on monolithic implementations, which minimizes the margin for improvement of a given circuit inserted on RF transceiver. Loop filter is one of synthesizer building blocks, which is designed for loop stability, settling time and locking range. The obtained values for the loop components may lead to a solution external to the synthesizer monolithic implementation. This enables the study, characterization and possible optimization of phase noise under different filter topologies.

The objective of this work is the characterization of phase noise in RF synthesizer based on the obtained modulated channel, minimizing its influence with loop filter topology optimization. The main contribution and novelty of this work consists on performing phase noise shaping without changing the LO characteristics. The validation of the proposed work is based on OFDM simulation scenario with configurable RF parameters [11], which enables the extraction of inter-carrier interference (ICI) for several loop filter topologies.

\section{Technological Innovation for Collective Awareness Systems}

Since every collective awareness system needs to be capable of linking several entities such objects, people or enterprises, it needs to connect to a physical device that enables the network interconnectivity between such elements. The communication system that supports collective awareness platforms must be connected to Internet in order to fulfill the information transaction. Assuming that every equipment establishes a wireless link, they need to have a RF unit to enable such communication. Therefore the study of optimization of radio systems contributes, indirectly, for the technological innovation in this area of study. 


\section{RF Charge-Pump Synthesizer Analysis}

Several synthesizer topologies can be found in literature [5], [8]. In this work the adopted topology is based on a charge-pump PLL with a divider in the feedback loop, as presented in Fig. 1.

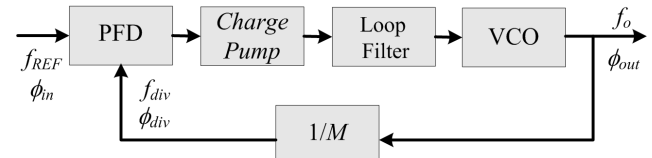

Fig. 1. Charge-pump synthesizer topology

Typical charge-pump synthesizer architecture uses a low frequency reference signal which phase $\phi_{i n}$ and frequency $f_{R E F}$ are compared with $\phi_{d i v}$ and $f_{d i v}$, obtained from the $M$ division of the output signal. The phase-frequency detector (PFD), together with the charge pump, produces an output voltage proportional to the phase and frequency difference between the reference signal and the feedback signal. The loop filter suppresses high frequency components from the output of the charge-pump and ensures loop stability, as presented in 3.1.1. The $M$ divider is configurable to obtain an output frequency multiple of the reference input signal. The output frequency $\left(f_{\text {out }}\right)$ is expressed by (1).

$$
f_{\text {out }}=M f_{\text {ref }} \text {. }
$$

Depending on the standards, the output signal must change in small frequency steps, typically hundreds of $\mathrm{kHz}$ in channels centered in the $\mathrm{GHz}$ range. These requirements make the synthesizer very sensitive to the building blocks impairments, namely LO phase noise.

Synthesizer transient response is a nonlinear process and is not addressed in this work. However, a linear approximation can be used, in order to obtain the trade-off building blocks design and their contribution to the loop stability [5]. Fig. 2a) shows the linear model of a synthesizer in lock conditions, with the transfer function for each block [5]. The obtained close loop transfer function is presented in expression (2).

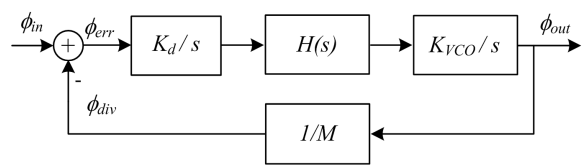

(a)

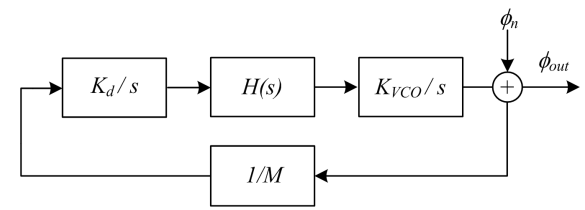

(b)

Fig. 2. Synthesizer linear model: (a) output to input; (b) output to VCO phase noise

$$
\frac{\phi_{\text {out }}(s)}{\phi_{\text {in }}(s)}=\frac{K_{d} \cdot K_{V C O} \cdot H(s)}{s^{2}+K_{d} \cdot K_{V C O} \cdot H(s) / M}
$$


If $\mathrm{H}(\mathrm{s})=1$, the closed loop reveals two conjugated imaginary poles, showing loop instability and its dependency on the loop filter design.

Classical approaches suggest that a zero must be added in the transfer function to avoid instability [5], [8]. However, these studies are not addressed on how the loop filter will influence the synthesizer phase noise.

\subsection{Charge-Pump Synthesizer Phase Noise}

Since the synthesizer needs to provide a very stable signal makes this topology susceptible to phase noise, which can be caused by the input reference signal and VCO [5]. These two influences must be studied independently, since their impact on the synthesizer is different [5].

The study of phase noise at input is presented in [5], [8]. The transfer function is similar to (2), where $\phi_{\text {in }}$ and $\phi_{\text {out }}$ reflects the phase variation of the input and output signal, respectively. The synthesizer itself, due to its negative feedback characteristic, can compensate the phase error. If the phase of the input signal has a slow variation with time, the synthesizer can track the signal. However, if the input phase has a fast variation with time, the synthesizer fails to track with the reference signal.

When VCO phase noise is taking into account, for simplicity of the loop analysis, it is assumed that the input excess phase is zero. The phase noise inherent to the VCO can be modulated as an additive noise [5]. Therefore, the loop is redrawn as depicted in Fig. 2b). Assuming that $\phi_{V C O}$ and $\phi_{i n}$ are uncorrelated, the superposition theorem can be applied, obtaining the transfer function (3), which presents the same poles of (2). The transfer function also contains two zeros at the origin, showing that synthesizer phase noise transfer function has a high pass characteristic.

$$
\frac{\phi_{\text {out }}(s)}{\phi_{V C O}(s)}=\frac{s^{2}}{s^{2}+K_{d} \cdot K_{V C O} \cdot H(s) / M}
$$

Classical synthesizer analysis addresses transfer function denominator into control theory form, where the loop natural frequency and damping factor is taking into account for the loop bandwidth and stability [5]. This is a useful and valid approach, however, when the loop filter topology complexity increases, an analytical expression, based on these parameters, is difficult to obtain. Therefore, numerical analysis is performed for ICI calculation as a function of the loop filter topology.

\subsubsection{Phase Noise Dependency on Loop Filter Design}

As depicted by (3) phase noise transfer depends on the loop filter topology, which will be shaped by the transfer function.

Table 1 shows the zero-pole values for the several loop filters used in this work. Filter $\mathrm{H}_{\mathrm{A}}(\mathrm{s})$ corresponds to the particular case when there is no loop filter in the synthesizer. The remaining loop parameters are given by $K_{V C O}=1 \mathrm{MHz} / \mathrm{V}$, $K_{d}=1 \mathrm{krad} / \mathrm{s}$ and $N=1000$. 
Table 1. Loop filter zero and pole values for each topology

\begin{tabular}{cccc}
\hline Loop Filter & Zero $[\mathrm{Hz}]$ & Pole $[\mathrm{Hz}]$ & Laplace Transfer Function \\
\hline $\mathrm{H}_{\mathrm{A}}(\mathrm{s})$ & - & - & 1 \\
$\mathrm{H}_{\mathrm{B}}(\mathrm{s})$ & - & 10000 & $\frac{\omega_{p}}{s+\omega_{p}}$ \\
$\mathrm{H}_{\mathrm{C}}(\mathrm{s})$ & 50 & 10000 & $\frac{\omega_{p}}{\omega_{z}} \cdot \frac{s+\omega_{z}}{s+\omega_{p}}$ \\
$\mathrm{H}_{\mathrm{D}}(\mathrm{s})$ & 50 & 100000 & \\
\hline
\end{tabular}

To minimize the synthesizer phase noise effect the different loop filter topologies presented in Table 1 are applied in expression (3). For each topology it is possible to infer the one that shows the minor overshoot, and consequently, evaluate the system stability and bandwidth.

Fig. 3a) and b) shows the transfer functions of the synthesizer and phase noise, respectively, for each loop filter presented in Table 1.

The obtained results shows that the synthesizer instability decreases with the increase of the loop filter complexity. The synthesizer transfer function and the respective phase noise transfer function, without the loop filter, presents an instability characteristic, since the poles are located on the imaginary axis.

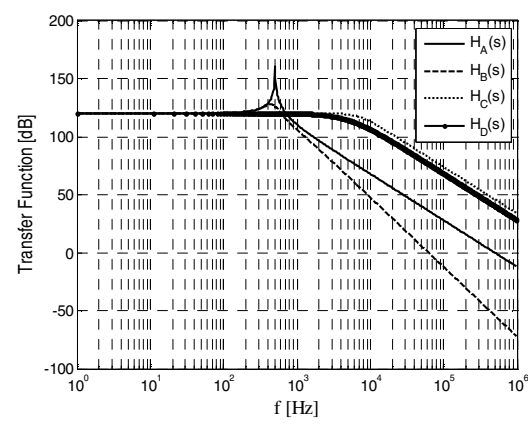

(a)

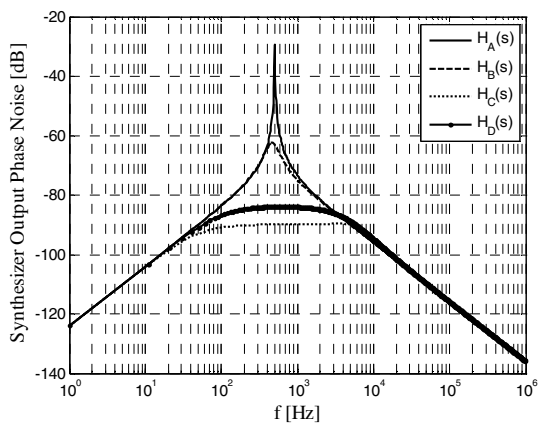

(c)

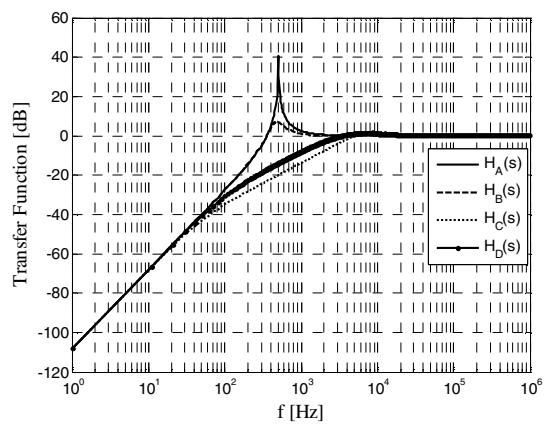

(b)

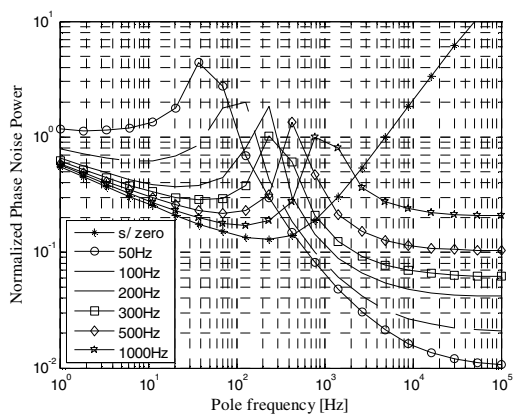

(d)

Fig. 3. (a) synthesizer transfer function; (b) phase noise transfer function; (c) synthesizer output phase noise (d) normalized integrated phase noise for frequency pole sweep 
Synthesizer output phase noise is shown in Fig. 3c). These values are obtained by multiplying (3) by the VCO integration function. As depicted, for each filter topology, the synthesizer transfer function presents different behaviors. To simplify the filter analysis, a numeric simulation is performed, based on frequency pole sweep. For each sweep, the integrated phase noise power is obtained and shown in Fig. 3d), regarding different zero values of the loop filter $\mathrm{H}_{\mathrm{C}}(\mathrm{s})$.

As expected, due to the loop instability, a filter with no zero is undesirable, since the integrated phase noise power will increase as the frequency pole increases. However, by increasing the frequency of the zero, for low pole values, the integrated phase noise power decreases, though these values do not allow the synthesizer loop to be stable. Increasing the pole frequency, the integrated phase noise increases until the frequency of the zero approximately, and then decreases. For the performed numeric simulations, the minimal integrated phase noise power corresponds to the zero at $50 \mathrm{~Hz}$. This evaluation will be taken in to account for the frequency conversion of the OFDM simulation scenario.

\section{$4 \quad$ Numerical Simulation of OFDM Inter-carrier Interference}

The developed OFDM simulation scenario is shown in Fig. 4. The transmitted data stream is mapped into 16-QAM with configurable symbol frequency. The IFFT and FFT use 64 points [11] and the cyclic prefix contains 16 extra samples per symbol. OFDM symbol windowing is a square-root raised cosine filter. For this work, the RF front-end emitter is considered ideal.

The receiver is composed by the RF front-end where, the LO used for downconversion, is modulated by a synthesizer with configurable noise mask presented in section 3.1.1. After the down-conversion, the obtained IQ signal is applied to OFDM RX block for CP removal, FFT operation and symbol de-mapping. ICI on the received signal is evaluated as a function of the used noise mask and consequently the loop filter topology, as expressed in section 3.1.1.

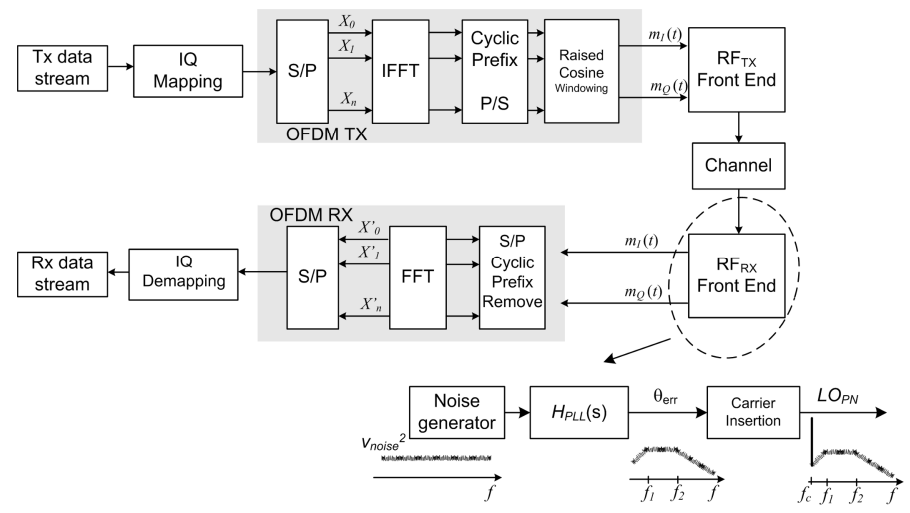

Fig. 4. OFDM block diagram MatLab/Simulink simulation 
The LO model, presented in Fig. 4, allows the configuration of the integrated phase noise power and bandwidth. Previous works show how phase noise power distribution affects single carrier [12] and OFDM [11] modulation schemes. In this work, it is possible to predict the noise power within an OFDM sub-channel under several phase noise masks for a specific synthesizer loop filter topology.

\subsection{Numerical Results}

Unlike single carrier systems [11], [12], OFDM spectral content does not present a direct relation with phase noise, due to information split through the several subchannels, the CP insertion and raised-cosine windowing. To obtain a significant result based on spectrum analysis, it is necessary to cancel the analyzed sub-channel, extracting the noise mean power within its bandwidth. This methodology allows the analysis of the adjacent sub-channels influence on the analyzed sub-channel.

Fig. 3c) and d) shows that loop filters $H_{C}(s)$ and $H_{D}(s)$ minimizes the synthesizer phase noise. Based on this results, $\mathrm{H}_{\mathrm{PLL}}(\mathrm{s})$ is modulated according to both phase noise mask depicted in Fig. 3c). Table 2 presents the synthesizer noise masks based on zero-pole mapping, where $\mathrm{H}_{\mathrm{PLLc}}(\mathrm{s}) \mathrm{H}_{\mathrm{PLLd}}(\mathrm{s})$ correspond to the output noise mask when the loop filters $\mathrm{H}_{\mathrm{C}}(\mathrm{s})$ and $\mathrm{H}_{\mathrm{D}}(\mathrm{s})$ are used.

Table 2. Zero-pole values for LO modulated noise

\begin{tabular}{cccc}
\hline Noise Mask & Zero $[\mathrm{Hz}]$ & Pole $1[\mathrm{~Hz}]$ & Pole 2 $[\mathrm{Hz}]$ \\
\hline $\mathrm{H}_{\text {PLLc }}(\mathrm{s})$ & 0 & 50 & 5000 \\
$\mathrm{H}_{\text {PLLc }}(\mathrm{s})$ & 0 & 100 & 2000 \\
\hline
\end{tabular}

As depicted in Fig. 5, OFDM ICI for the same number of active sub-channels, presents a lower value for the phase noise mask $\mathrm{H}_{\mathrm{PLLc}}(\mathrm{s})$ comparing with $\mathrm{H}_{\mathrm{PLLd}}(\mathrm{s})$, which confirms the results presented in Fig. 3c) and d).

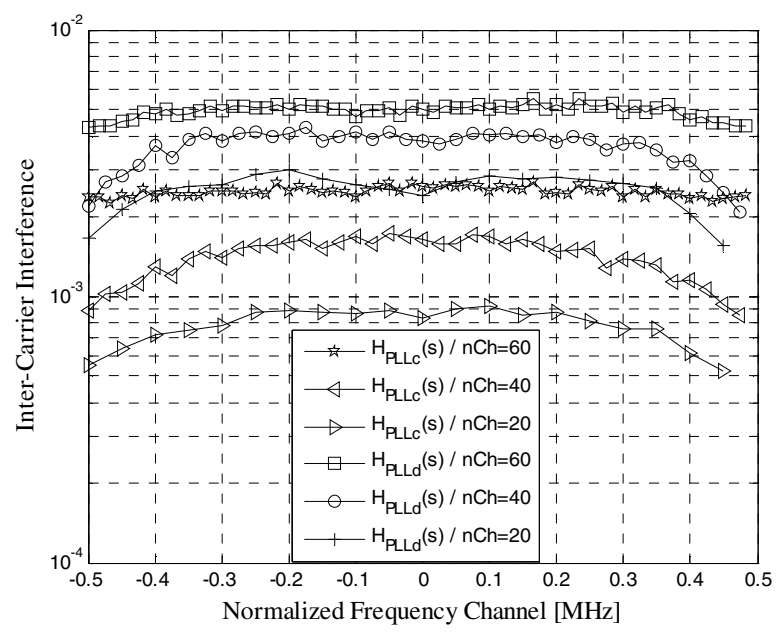

Fig. 5. ICI for different loop filter topologies 


\section{Conclusions}

The influence of the synthesizer loop filter design on the phase noise of a charge pump RF synthesizer was described. Different passive filter designs were studied in order to obtain a topology that minimizes the synthesizer phase noise. The validation method was performed with a developed OFDM simulation model that allows the synthesizer modeling based on a specific noise mask.

The obtained results show that is possible to minimize OFDM ICI by changing the synthesizer loop filter topology, namely the poles and zeros values. This is an interactive process since the minimal noise value may drive synthesizer loop into instability. OFDM ICI, besides the loop filter topology, also depends on the number of active sub-channels. Therefore, besides the loop filter design, ICI can be optimized in function of the active sub-channels. This feature revels to be and important trade off factor when the optimization of phase noise is take into account.

As future work, the proposed method will be experimentally validated with a radio transceiver.

\section{References}

1. IEEE Standard for Wireless LAN Medium Access Control (MAC) and Physical Layer (PHY) Specifications: High Speed Physical Layer Extension in the 2.4GHz, IEEE Standard 802.11b-1999

2. IEEE 802.16. IEEE Recommended Practice for Local and metropolitan area networks Coexistence of Fixed Broadband Wireless Access Systems

3. Armstrong, J., et al.: OFDM for Optical Communications. Journal of Lightwave Technology 27(3) (February 2009)

4. Armanda, A.G.: Understanding the effects of phase noise in orthogonal frequency division multiplexing (OFDM). IEEE Transactions on Broadcasting 47(2) (June 2001)

5. Razzavi, B.: RF Microelectronics. Prentice Hall (1998)

6. Leeson, D.B.: A Simple Model of Feedback Oscillator Noise Spectrum. Proceedings of the IEEE 54(2), 329-330 (1966)

7. Lee, T.H., Hajimiri, A.: Oscillator Phase Noise: A Tutorial. IEEE Journal of Solid-State Circuits 35(3), 326-336 (2000)

8. Mathecken, P., et al.: Characterization of OFDM Radio Link Under PLL-Based Oscillator Phase Noise and Multipath Fading Channel. IEEE Transactions on Communications 60(6) (June 2012)

9. Petrovic, D., et al.: Phase Noise Influence on Bit Error Rate, Cut-off Rate and Capacity of M-QAM OFDM Signaling. In: Proc. Intl. OFDM Workshop of M-QAM OFDM Signaling, InOWo (2002)

10. Tchamov, N.N., et al.: VCO phase noise trade-offs in PLL design for DVB-T/H receivers. In: 16th IEEE International Conference on Electronics, Circuits, and Systems-ICECS, pp. 527-530 (December 2009)

11. Fialho, V., Fortes, F., Vieira, M.: Local Oscillator Phase Noise Influence on Single Carrier and OFDM Modulations. In: Camarinha-Matos, L.M., Tomic, S., Graça, P. (eds.) DoCEIS 2013. IFIP AICT, vol. 394, pp. 513-520. Springer, Heidelberg (2013)

12. Fialho, V., Fortes, F., Vieira, M.: Test Setup for Error Vector Magnitude Measurement on WLAN Transceivers. In: 19th IEEE International Conference on Electronics, Circuits, and Systems-ICECS, pp. 917-920 (December 2012) 